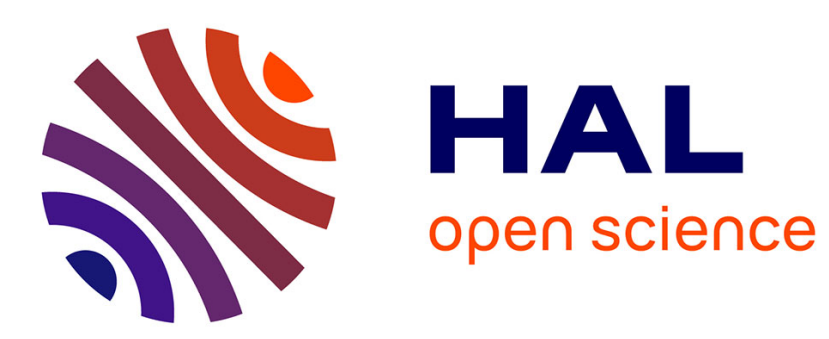

\title{
Entre travail et famille: à propos de l'évolution du modèle suédois
}

\author{
Anne-Marie Daune-Richard, Anita Nyberg
}

\section{To cite this version:}

Anne-Marie Daune-Richard, Anita Nyberg. Entre travail et famille: à propos de l'évolution du modèle suédois. Revue française des affaires sociales, 2003, 4 numéro spécial, pp.515-527. halshs-03351785

\section{HAL Id: halshs-03351785 \\ https://shs.hal.science/halshs-03351785}

Submitted on 22 Sep 2021

HAL is a multi-disciplinary open access archive for the deposit and dissemination of scientific research documents, whether they are published or not. The documents may come from teaching and research institutions in France or abroad, or from public or private research centers.
L'archive ouverte pluridisciplinaire HAL, est destinée au dépôt et à la diffusion de documents scientifiques de niveau recherche, publiés ou non, émanant des établissements d'enseignement et de recherche français ou étrangers, des laboratoires publics ou privés. 


\title{
Entre travail et famille : à propos de l'évolution du modèle suédois
}

\author{
Anne-Marie Daune-Richard et Anita Nyberg*
}

La relation entre travail et famille, en particulier à partir de la question du « travail centré sur autrui » (Letablier, 2001) - ce que les anglophones appellent le « care »- apparaît aujourd'hui comme un enjeu central de la (re)définition de la «pleine » citoyenneté.

Partant de la proposition de T.H. Marshall ${ }^{1}$ qui voyait dans les droits sociaux la troisième et dernière dimension - après les droits civils et civiques - de la citoyenneté moderne, les recherches féministes ont montré que les femmes n'y avaient accédé que tardivement et incomplètement (cf. entre autres : Orloff, 1993 ; O’Connor, 1993 et 1996 ; Sainsbury, 1994 ; Walby, 1994). Dans le modèle d'individualisation de la société salariale, les droits sociaux sont assis sur l'emploi : la pleine citoyenneté est celle du travailleur. Or les charges domestiques et parentales qui incombent majoritairement aux femmes limitent leur accès au marché du travail et aux droits qui y sont associés. Le citoyen de nos sociétés modernes est ainsi a priori un homme (Daune-Richard, 1997) voire un pater familias (Verjus, 2002).

La Suède fait figure de pays pilote dans la mise en œuvre d'une relation entre travail et droits sociaux qui autorise et développe, pour toutes et tous, l'accès à la pleine individualité, condition de la citoyenneté. En particulier, les charges éducatives y ont été considérées comme une responsabilité partagée entre les pères et les mères d'un côté, entre les parents et l'État de l'autre, ce dernier étant le garant du droit de l'enfant au bien-être et à l'éducation.

La grave crise économique qu'a connue la Suède dans les années quatre-vingt-dix s'est traduite par d'importantes restrictions budgétaires, en particulier dans le domaine de la protection sociale. Dans un contexte international où la crise des systèmes de protection sociale conduit à des recompositions des États providence qui se sont généralisés dans l'immédiat après-guerre, on questionnera ici la résistance du modèle suédois de prise en charge des jeunes enfants à cette crise.

\footnotetext{
* Anne-Marie Daune-Richard : sociologue, chargée de recherche au CNRS (Laboratoire d'économie et sociologie du travail). Anita Nyberg : économiste, professeure à l'Institut pour la vie de travail de Stockholm.

1 Dans Citizenship and Social Class (Marshall T.H., 1950).
} 


\section{- La résistance du modèle suédois de prise en charge de l'enfance}

Le modèle suédois de prise en charge de la petite enfance se met en place dans les années soixante-dix au carrefour de deux «référentiels » de l'action publique ${ }^{1}$ (Daune-Richard, 1998a et 1999).

D'un côté, un référentiel dans lequel, pour chacun et chacune, travailler et prendre soin de ses enfants fait partie de ses droits et devoirs. On n'est pas ici dans une approche en termes de « conciliation » entre les charges professionnelles et familiales, ni même dans une quête des «droits des femmes » mais bien dans un référentiel d'égalité des droits et obligations au regard des engagements professionnels et familiaux. Tous et toutes doivent être - et pouvoir être - des individus, autonomes et responsables. C'est la pleine individualisation de la société qui est visée ici et donc la généralisation aux femmes de la «pleine » citoyenneté.

D'un autre côté, l'enfant suédois est, dès le plus jeune âge, considéré comme un citoyen qui, s'il n'est pas en mesure d'exercer des devoirs, a des droits : droit au bien-être, à être entouré et éduqué, l'État providence étant le garant de ce bien-être comme de celui de tous les citoyens. En développant une prise en charge de l'enfant extérieure à la famille, il le fait dans les principes qui sont les siens : service public, universel, répondant à des normes de qualité exigeantes, visant à égaliser les conditions d'éducation par-delà la diversité des dotations familiales.

Comment ce modèle de prise en charge de la petite enfance a-t-il traversé l'épreuve de la crise des années quatre-vingt-dix?

\section{Du point de vue de l'égalité des sexes}

\section{La participation des femmes au marché du travail est toujours très élevée}

La crise de l'emploi touche violemment la Suède au début de la décennie : le taux de chômage s'envole, passant de moins de $2 \%$ à plus de $9 \%$ entre 1990 et 1994. Cependant, tout au long de cette décennie, les hommes sont plus touchés que les femmes car les secteurs masculins sont ceux qui connaissent le plus de réduction d'emplois : ainsi, en 1994, le taux de chômage des hommes atteint $9,1 \%$, celui des femmes $6,9 \%$.

D'une façon générale entre 1990 et aujourd'hui, l'activité et l'emploi tendent à diminuer pour les deux sexes (cf. tableau 1).

1 «Le référentiel d'une politique est constitué d'un ensemble de normes prescriptives qui donnent un sens à un programme politique en définissant des critères de choix et des modes de désignation des objectifs » (Muller P., 1994, p. 43). 
Tableau 1 : Taux d'activité, d'emploi et de chômage. 16-64 ans

\begin{tabular}{|l|c|c|c|c|}
\hline \multirow{2}{*}{} & \multicolumn{2}{|c|}{ Hommes (en \%) } & \multicolumn{2}{c|}{ Femmes (en \%) } \\
\cline { 2 - 5 } & $\mathbf{1 9 9 0}$ & $\mathbf{2 0 0 2}$ & $\mathbf{1 9 9 0}$ & $\mathbf{2 0 0 2}$ \\
\hline Taux d'activité & 86,6 & 79,8 & 82,3 & 76,1 \\
Taux d'emploi & 85,2 & 76,3 & 81,0 & 73,6 \\
Taux de chômage & 1,7 & 4,4 & 1,6 & 3,6 \\
\hline
\end{tabular}

Source : Labour Force Surveys, Statistics Sweden.

Si l'on considère les parents d'enfants d'âge préscolaire (moins de 7 ans) on constate (cf. tableau 2) cependant que cette tendance à la diminution est nettement plus accentuée pour les mères que pour les pères. La différence est particulièrement forte pour les taux d'emploi, ce qu'il faut rapporter au fait que les congés parentaux sont plus utilisés par les mères que par les pères (ce que nous verrons plus loin). Or les personnes en congé parental sont, en Suède, comptées dans la population active.

Tableau 2 : Taux d'activité, d'emploi et de chômage des parents d'un enfant d'âge préscolaire

\begin{tabular}{|l|c|c|c|c|}
\hline & \multicolumn{2}{|c|}{ Pères (en \%) } & \multicolumn{2}{c|}{ Mères (en \%) } \\
\cline { 2 - 5 } & $\mathbf{1 9 9 0}$ & $\mathbf{2 0 0 2}$ & $\mathbf{1 9 9 0}$ & $\mathbf{2 0 0 2}$ \\
\hline Taux d'activité & 96,7 & 93,5 & 86,3 & 80,7 \\
Taux d'emploi & 95,6 & 90,9 & 85,0 & 77,5 \\
Taux de chômage & 1,0 & 2,6 & 1,2 & 3,2 \\
\hline
\end{tabular}

Source : Labour Force Surveys, Statistics Sweden.

Si l'on se tourne maintenant du côté du temps consacré au travail professionnel, l'évolution va dans le sens d'un rapprochement entre les sexes. En 1990, les hommes en emploi y consacraient en moyenne 40,3 heures (effectives) et les femmes 32,6 heures. En 2002, les chiffres correspondant sont de 39,4 et 33,4 heures. D'une façon générale, la part des femmes travaillant à plein temps s'est fortement accrue, en particulier à partir de la fin de la décennie : $59 \%$ en 1990, $61 \%$ en 1996, $65 \%$ en 2000 (cf. tableau 3).

Dans le même temps, le travail à temps partiel a diminué chez les femmes et légèrement augmenté chez les hommes. Il ne faut pas oublier cependant que cette forme d'emploi est fortement liée en Suède aux formes légales d'absentéisme - et, dans ce cadre, financièrement compensée - et d'aménagement du temps de travail : c'est le cas ; par exemple ; des congés parentaux (cf. Daune-Richard, 1993 et 1998b). En ce sens, le travail à temps partiel ne peut être considéré comme une forme de régulation du chômage : $23 \%$ des hommes et $22 \%$ des femmes employés à temps partiel se déclaraient l'an dernier en situation de sous-emploi. 
Tableau 3 : Durée du travail hebdomadaire des salariés

\begin{tabular}{|l|r|r|r|r|}
\hline \multirow{2}{*}{} & \multicolumn{2}{|c|}{ Hommes } & \multicolumn{2}{c|}{ Femmes } \\
\cline { 2 - 5 } & $\mathbf{1 9 9 0}$ & $\mathbf{2 0 0 2}$ & $\mathbf{1 9 9 0}$ & $\mathbf{2 0 0 2}$ \\
\hline 35 heures et plus & $92,5 \%$ & $90,2 \%$ & $59,5 \%$ & $65,5 \%$ \\
20-34 heures & $5,0 \%$ & $6,2 \%$ & $34,7 \%$ & $27,6 \%$ \\
$1-19$ heures & $2,0 \%$ & $3,2 \%$ & $5,6 \%$ & $6,4 \%$ \\
\hline
\end{tabular}

Source : Labour Force Surveys, Statistics Sweden.

Cette forme d'emploi est néanmoins très liée pour les femmes à la présence d'enfants, ce qui ne se vérifie pas chez les hommes. Cependant, pendant la dernière décennie, le travail à temps partiel a fortement décru chez les mères (cf. tableau 4).

Tableau 4 : Emploi à temps partiel des mères selon l'âge du plus jeune enfant ${ }^{1}$

\begin{tabular}{|l|c|c|}
\hline & $\mathbf{1 9 9 0}$ & $\mathbf{2 0 0 1}$ \\
\hline $1-2$ ans & $59,5 \%$ & $47,0 \%$ \\
$3-6$ ans & $60,0 \%$ & $43,6 \%$ \\
$7-10$ ans & $52,4 \%$ & $37,8 \%$ \\
$11-16$ ans & $40,7 \%$ & $29,6 \%$ \\
\hline
\end{tabular}

1 Avant un an, les taux d'emploi à temps partiel sont encore plus bas (27\% des mères en 2001) mais, à cet âge, la plupart des enfants sont gardés à la maison par un parent en congé parental et pratiquement aucun n'est recensé dans un mode public de garde.

Source : Labour Force Surveys, Statistics Sweden.

\section{L'égalité devant les charges familiales progresse}

D’une façon générale le « travail total » (professionnel et domestique) est mieux réparti entre les femmes et les hommes en Suède qu'en France. Le partage demeure néanmoins tout à fait inégalitaire même si la progression est réelle (cf. Anxo, Flood et Kocoglu, 2002). Entre 1984 et $1993^{1}$, la part des femmes dans les temps consacrés par les ménages suédois aux différentes activités, est passée de 37 à $40 \%$ pour le travail professionnel, de 64 à $59 \%$ pour le travail domestique et de 59 à $60 \%$ pour les « soins aux tiers » (idem, p. 131).

Parallèlement, pendant la dernière décennie les hommes ont accru leur participation aux congés parentaux (cf. tableau 5). Des modifications intervenues dans la réglementation des congés parentaux ont probablement influencé cette évolution. En particulier, l'instauration de jours non transférables entre parents. En 1990, les droits aux 450 jours de congé étaient ouverts indifféremment à l'un ou l'autre des deux parents. En 1995 a été

1 Dates des deux dernières enquêtes suédoises sur les emplois du temps (Household Market and Non-Market Activities) étudiées par les auteurs. 
introduit un mois « réservé » à l'un des deux (immédiatement surnommé le « mois du père »). En 2002, un deuxième mois « réservé » était institué en même temps que les droits étaient portés à 480 jours de congé. À partir du milieu de la décennie, la part des pères en congé augmente sensiblement mais leur contribution en part des jours de congé pris progresse beaucoup plus lentement.

Tableau 5 : Part des pères dans les bénéficiaires des congés parentaux indemnisés

\begin{tabular}{|l|c|c|}
\hline & $\begin{array}{c}\text { Part des pères parmi les } \\
\text { parents en congé parental } \\
\text { (en \%) }\end{array}$ & $\begin{array}{c}\text { Part des jours de congé } \\
\text { pris par les pères } \\
\text { (en \%) }\end{array}$ \\
\hline 1990 & 26,1 & 8,8 \\
1995 & 28,5 & 9,2 \\
1998 & 32,4 & 10,4 \\
2000 & 37,7 & 12,4 \\
2001 & 40,0 & 13,8 \\
2002 & non disponible & 15,0 \\
\hline
\end{tabular}

Source : Sweden RFV (Rikförsäkningverket).

\section{L'autonomie financière des femmes grandit}

Une analyse des enquêtes suédoises sur la répartition des revenus de 1975 et $1994{ }^{1}$ (Nyberg, 2000) montre que l'État providence contribue sensiblement à réduire la disparité des revenus entre femmes et hommes. Ainsi, en début de période le revenu moyen qu'elles tiraient de l'emploi comptait pour $43 \%$ de celui des hommes ; mais une fois pris en compte les transferts sociaux, leur revenu disponible s'élevait à $57 \%$ de celui des hommes. En 1994, les chiffres correspondant étaient respectivement de $65 \%$ et $78 \%$ (Nyberg, 2000, p. 53) : l'autonomie financière des femmes grandit en même temps que leur contribution au travail rémunéré.

En considérant maintenant les revenus à l'intérieur des ménages, l'évolution marquante est que la femme apportait $25 \%$ des revenus de l'emploi entrant dans le ménage en 1975, $40 \%$ en fin de période. Après transferts monétaires et imposition, la contribution féminine au revenu disponible du ménage était de $31 \%$ en 1975 et de $40 \%$ en 1994 (id., p. 60). Elle est devenue identique à la part qu'elles apportent dans les revenus de l'emploi.

Au total, dans la période considérée, les sources des revenus (rémunération du travail ou prestations sociales) des hommes et des femmes se sont équilibrées (cf. tableau 6).

1 Seules dates disponibles. 
Tableau 6 : Part du revenu brut moyen en provenance respectivement de l'emploi et de l'État providence. Hommes et femmes : 18-64 ans

\begin{tabular}{|l|c|c|c|c|}
\hline \multirow{2}{*}{} & \multicolumn{2}{|c|}{ Hommes (en \%) } & \multicolumn{2}{c|}{ Femmes (en \%) } \\
\cline { 2 - 5 } & $\mathbf{1 9 7 5}$ & $\mathbf{1 9 9 4}$ & $\mathbf{1 9 7 5}$ & $\mathbf{1 9 9 4}$ \\
\hline Emploi & 91 & 80 & 80 & 72 \\
État providence & 9 & 20 & 20 & 28 \\
\hline Total & 100 & 100 & 100 & 100 \\
\hline
\end{tabular}

Source : Institut de la statistique suédoise, Enquête sur la répartition des revenus (Nyberg, 2000, p. 51).

Et ceci se vérifie aussi lorsqu'on ne considère que les ménages ayant des enfants à charge (Nyberg, 2002).

\section{Du point de vue des exigences de qualité et d'universalité du service public}

Le référentiel suédois de prise en charge de la petite enfance veut qu'un service public de qualité vienne appuyer les parents dans leurs responsabilités familiales tout en dispensant des apports éducatifs destinés à promouvoir une égalisation des chances entre enfants issus de milieux socioculturels différents. En ce sens, les référents du modèle sont : l'accessibilité voire l'universalité, la qualité des services, le contrôle de l'État providence garant du bien-être collectif ${ }^{1}$.

\section{Un grand service public...}

La dernière décennie ayant été marquée par le contrôle et la réduction des dépenses publiques, le développement d'un grand service public de prise en charge des jeunes enfants était mis à l'épreuve. Si l'indemnisation des congés (parentaux mais pas seulement eux) a été réduite de $85 \%$ à $75 \%$ du salaire (puis remontée à $80 \%$ en 1998), l'effort consacré aux services de l'enfance a été maintenu en dépit des difficultés budgétaires.

D'un côté, le nombre de places offertes par le service public a augmenté pendant la décennie et, de l'autre, la priorité a été donnée aux crèches collectives tandis que les places en crèches familiales diminuaient fortement. Les premières accueillaient 267000 enfants en 1990 et 315000 en 2001 ; les secondes 156000 enfants et moins de 50000 respectivement à ces mêmes dates. Au total la part des enfants accueillis dans les crèches n'a cessé de croître pendant la période (cf. tableau 7).

1 Un précédent bilan du modèle suédois de prise en charge de la petite enfance a été fait par C. Berqvist et A. Nyberg (2002). Il est en partie repris ici et actualisé. 
Tableau 7 : Part des enfants accueillis dans un établissement public (crèches collectives et familiales) par groupe d'âge (en \%)

\begin{tabular}{|c|c|c|c|}
\hline & $\mathbf{0 - 2}$ ans & 3-6 ans & 0-6 ans \\
\hline 1990 & 29 & 64 & 48 \\
1994 & 32 & 73 & 55 \\
1995 & 37 & 74 & 59 \\
1996 & 40 & 83 & 66 \\
1997 & 41 & 84 & 68 \\
1998 & 42 & 79 & 66 \\
1999 & 40 & 82 & 66 \\
2000 & 60 & 86 & 68 \\
\hline
\end{tabular}

NB : les enfants fréquentant la classe préscolaire, créée en 1999 pour les moins de 7 ans, ne sont pas comptés dans les tableaux présentés ici sauf s'ils sont en même temps accueillis en crèche.

Source : Statistical Yearbook.

Parallèlement, les modes de garde privés se sont développés mais restent nettement minoritaires : $5 \%$ des enfants fréquentaient ce type d'établissement en 1990, $17 \%$ aujourd'hui. Les établissements sont subventionnés par les budgets publics et sont tenus de répondre à des normes équivalentes à celles des établissements publics. Ils sont surtout présents dans les grandes agglomérations urbaines et, pour la moitié d'entre eux, ce sont des coopératives de parents.

\section{De qualité ?}

La qualité d'un service d'accueil des jeunes enfants n'est pas aisée à mesurer. Un des moyens d'approcher la question est d'étudier les ressources affectées à ce service. On observe, ici, l'évolution du nombre d'enfants sous la responsabilité d'un éducateur. Il a augmenté tout au long des années quatre-vingt-dix et s'est réduit en fin de période : 4,2 enfants par éducateur en $1990,5,2$ en $1994,5,7$ en $1998,5,4$ en 2000 et 5,3 en $2002^{1}$.

Cependant, dans le même temps la formation des personnels s'est considérablement élevée. Deux types de professions exercent dans la prise en charge de la petite enfance : des assistantes maternelles qualifiées et des institutrices spécialisées détentrices d'un diplôme universitaire. Jusqu'au début des années quatre-vingt-dix, les premières étaient majoritaires tandis qu'aujourd'hui les secondes forment le groupe le plus important. Parallèlement, pendant la dernière décennie, l'organisation du travail dans le service public de l'enfance s'est modifiée et a été décentralisée. On peut ainsi supposer que, en terme de qualité du service, l'augmentation des effectifs d'enfants affectés à chaque éducateur a été compensée par l'élévation de la qualification des personnels et par la réorganisation du travail.

1 Source : 1990-1993 - VälfärdsBulletinen nr 6, 1994 ; 1994-1999 - Skolverket, 2000b : Tabell 1.7A. 


\section{Plus universel et égalitaire?}

En 1985, la loi suédoise a institué un droit d'accueil pour chaque enfant à partir de l'âge d'un an. Cependant, dans la réalité, ce droit a été longtemps limité aux enfants dont les parents avaient un emploi ou poursuivaient des études et à ceux ayant des besoins spécifiques (handicap par exemple). De plus, la hausse de la fécondité à la fin des années quatre-vingt a induit une pression forte sur la demande et créé des listes d'attente. Enfin, dans les années quatre-vingt-dix, la montée du chômage a révélé un problème pour les parents dans cette situation qui ne pouvaient obtenir de place pour leurs enfants.

En 1995, une nouvelle loi spécifie que les municipalités doivent répondre aux demandes dans des délais « raisonnables ». Et en 2001, celles-ci se voient dans l'obligation d'offrir au moins 15 heures par semaine d'accueil et d'activités préscolaires aux enfants de parents au chômage. En janvier 2002, cette obligation est étendue aux enfants dont les parents sont en congé parental avec un autre enfant. Enfin, depuis janvier 2003, elles sont tenues d'offrir 15 heures d'activités préscolaires, gratuites, pour les enfants de 4-5 ans. Parallèlement, la possibilité d'accéder à une classe « préscolaire » destinée aux enfants de 6 ans (l'école primaire commençant à 7 ans) a été ouverte en 1999 (mais semble peu utilisée).

Un autre point crucial pour l'accessibilité du système d'accueil réside dans la charge financière qu'il représente pour les familles. Tout au long de la décennie précédente, la part du coût revenant aux parents n'a cessé d'augmenter : en 1990, leur contribution couvrait $10 \%$ du coût total, contre $19 \%$ en 2000 (Skolverket, 2001, p. 25). De plus, les variations de prix demandés par les municipalités étaient très importantes, introduisant des inégalités considérables entre parents. Un plafonnement des tarifs a été institué en 2002. D’une part, le prix payé par les parents est désormais limité à $3 \%$ de leurs revenus avant impôt pour un enfant, $2 \%$ pour le deuxième, $1 \%$ pour le troisième. D'autre part, un plafond a été introduit (environ 114 euros pour le premier enfant, dégressif pour les suivants) ${ }^{1}$.

Au total, on est fondé à conclure que la société suédoise a fait des efforts considérables pour développer et faciliter l'accès de tous les enfants, indépendamment du statut socioprofessionnel de leurs parents, à son service public d'accueil. Deux mesures introduisent cependant un bémol dans ce diagnostic : la part du coût moyen des services payants laissée à la charge des parents qui a presque doublé et l'introduction d'un plafonnement des tarifs qui, finalement, favorise les familles plus aisées.

1 Pour que ces mesures ne risquent pas d'entraîner une baisse de la qualité du service offert par les municipalités, celles-ci se voient attribuer par l'État des subventions destinées à compenser le manque à gagner. 


\section{Les limites du modèle}

On discutera ici les limites du modèle du point de vue des rapports entre les sexes.

\section{Un modèle inachevé}

Le référentiel veut que les hommes et les femmes soient égaux devant les charges professionnelles et familiales. Sauf handicap ou difficulté particulière, il est de la responsabilité de chaque citoyen de contribuer à la reproduction sociale par son apport en travail à la richesse nationale et par l'accomplissement de ses devoirs envers autrui, ses enfants en particulier. Le modèle demande donc aux femmes d'être plus présentes dans le monde du travail professionnel et aux hommes d'être plus actifs dans l'espace familial.

Il est évident que depuis les années soixante-dix, qui ont vu la mise en œuvre de ce référentiel dans les politiques publiques, les évolutions sont allées clairement - et sans retour - dans ce sens. Mais il est non moins évident que le modèle n'est pas pleinement accompli : si l'on considère la participation au travail rémunéré, la famille suédoise tend à se construire sur la norme d' « un apporteur trois quarts », et, on l'a vu, le travail domestique et parental reste inégalement réparti entre hommes et femmes.

Au milieu de la dernière décennie, le gouvernement suédois a commandé un «Livre blanc » sur les inégalités entre les sexes ${ }^{1}$. Une étude menée dans ce cadre par G. Arhne et C. Roman (2000) met ainsi en évidence l'asymétrie qui continue de régner dans l'organisation des couples suédois puisque, dans la grande majorité d'entre eux, la femme consacre nettement plus de temps que l'homme au travail domestique et nettement moins de temps au travail rémunéré. Seule une petite minorité, le plus souvent sans enfant, partage équitablement le travail domestique; tandis que dans une autre minorité, un peu plus importante que la première, la femme fait tout ou pratiquement tout ce travail. Les auteur(e)s soulignent ainsi la coexistence de normes sociales contradictoires. D'un côté, un principe largement partagé par tous et toutes : celui de l'égalité des hommes et des femmes devant le travail, rémunéré et non rémunéré, et donc l'obligation, pour les femmes, de contribuer au budget familial et, pour les hommes, de contribuer au travail de la maison. De l'autre, une pratique qui admet l'asymétrie de la participation de chacun et chacune au «travail total » (Glucksman, 1997) assumé par la famille. Cette asymétrie autorise les femmes à ne pas s'inscrire à temps plein dans le travail rémunéré, alors que le travail à temps partiel doit rester une exception pour

1 Le numéro 27 des Cahiers du Genre (2000) est entièrement consacré à ce Livre blanc. 
les hommes : elles conservent ainsi la responsabilité du travail domestique, les hommes étant tenus d'y « contribuer».

Étudiant l'assurance parentale, Sara Brachet (2001) aboutit à des conclusions similaires. Elle montre la contradiction entre, d'un côté, le référentiel des politiques publiques qui affirme l'égalité des parents devant les engagements professionnels et parentaux et, de l'autre, une conception « experte », largement partagée par le sens commun, qui situe différemment les hommes et les femmes au regard de la parentalité. Mettant en effet l'accent sur l'importance de la présence des parents pour le bien-être des enfants avant un an et sur l'importance de l'allaitement pour sa santé ${ }^{1}$, les institutions et les experts définissent une norme fortement opératoire puisque, avant un an, les structures éducatives offrent très peu de places et les enfants sont gardés à la maison par un parent en congé parental... le plus souvent la mère. Pour Sara Brachet, la maternité serait ainsi « incontournable » tandis que la paternité serait « négociable » et aurait plus à voir avec la découverte éducative qu'avec l'astreinte des soins quotidiens.

\section{Un simple problème de transition?}

L'inachèvement du modèle suédois de rapport «hommes-femmes-travail-citoyenneté » peut être interprété en termes de transition entre l'inactivité traditionnelle des mères de famille et une activité soutenue et impulsée par les institutions politiques et économiques (Anxo et Daune-Richard, 1991). Les difficultés économiques auraient pu entraîner une pause voire

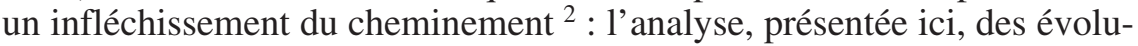
tions intervenues depuis le début des années quatre-vingt-dix plaide en faveur de la « résistance » du modèle à ces difficultés.

Il n'en demeure pas moins que, dans cette évolution sociopolitique, les hommes n'ont pas fait un mouvement vers les espaces du domestique et du parental à la mesure de celui que les femmes ont fait vers le professionnel. D'un côté, on l'a vu, les charges familiales restent, dans les pratiques mais aussi dans les représentations sociales, associées au féminin. De l'autre, dans l'emploi, les femmes restent concentrées dans les secteurs qui leur sont traditionnellement réservés. Et malgré un mouvement important de déspécialisation de l'emploi, tant féminin que masculin, la ségrégation sexuée du marché du travail suédois demeure une des plus élevée des pays occidentaux (Melkas et Anker, 1997 ; Persson et Wadensjö, 2000). Or la valeur du travail dans les professions féminines associées aux services aux personnes, au "care », est traditionnellement moindre que dans celles associées au technique et au masculin (Daune-Richard, 1998c). Ainsi, dans la Suède des années

1 À six mois, $75 \%$ des enfants suédois sont encore allaités.

2 Ce qu'on peut analyser en France, cf. Jenson J. et Sineau M., 1998 ; Daune-Richard A.-M., (à paraître). 
quatre-vingt-dix, les salaires ont baissé dans les secteurs de la santé et de l'éducation relativement aux autres (Persson et Wadensjö, 2000). Et c'est le fait que l'éventail des salaires est moins ouvert en Suède qu'ailleurs, combiné à l'élévation du niveau de formation des femmes, qui réduit le « coût » salarial de leur spécialisation professionnelle.

En ce sens, les critiques que Jane Lewis (2002) adresse aux recompositions des États providence européens ne s'appliquent pas réellement à la Suède. Analysant le mouvement actuel qui veut étendre aux femmes le modèle du citoyen-travailleur, historiquement réservé aux hommes dans la société salariale, elle souligne la nécessité avant de généraliser ce nouveau modèle de famille, de mettre la question du « care » au centre des analyses et préoccupations des politiques publiques. Arguant du fait que l'engagement des femmes dans l'emploi n'entraîne pas automatiquement un meilleur partage des charges familiales, ni une revalorisation des emplois de services aux personnes, elle affirme que la question ne se réduit pas à un simple problème de « conciliation » destinée à faciliter l'accès des mères au marché du travail. Or ce que l'on constate dans le cas suédois, c'est une vision politique beaucoup plus large qui, depuis trois décennies, insiste sur la double responsabilité de chacun, homme ou femme, devant l'emploi et les charges familiales : le référentiel ne se limite ni à un problème de conciliation ni à une problématique en termes de «droits des (seules) femmes ». Il n'en demeure pas moins que la continuité des politiques publiques menées dans ce sens peine à engager pleinement les hommes dans les charges familiales.

On touche ici à la difficulté soulignée par Knijn T. et Kremer M. (1997), à intégrer le «care », le travail centré sur autrui, dans les droits et devoirs du citoyen. Tout se passe comme si, en Suède, différence se conjuguait moins qu'ailleurs avec hiérarchie sociale : cela se vérifie, en particulier mais pas seulement, pour les différences de sexe. Ainsi, jusqu'à aujourd'hui, les soins aux autres restent largement une affaire de femmes et, contrairement au référentiel mis en œuvre depuis plusieurs décennies, le travail du " care » demeure inégalement réparti entre les hommes et les femmes. En même temps, la société suédoise a été labellisée, en 1995, par l’ONU comme « la plus égalitaire du monde».

On peut ainsi se demander si le modèle suédois ne bute pas ici sur une limite plus radicale qui dépasserait un « simple » problème de transition. Si cela se vérifiait, il serait du plus haut intérêt d'observer si et comment cette limite radicale peut être affrontée, voire surmontée. 


\section{Références bibliographiques}

ANXO D. et DAUNE-RICHARD A.-M., (1991), « La place relative des hommes et des femmes sur le marché du travail : une comparaison France-Suède », Travail et Emploi, 47.

ANXO D., FLOOD L. et KOCOGLU Y., (2002), « Offre de travail et répartition des activités domestiques et parentales au sein du couple : une comparaison entre la France et la Suède », Économie et Statistique, 352-353.

ARHNE G. et ROMAN C., (2000), « Travail domestique et rapports de pouvoir entre les sexes », Cahiers du Genre, 27.

BERGQVIST Ch. and NYBERG A., (2002), «Welfare State Restructuring and Child Care in Sweden », in S. Michel and R. Mahon, (ed.), Child Care policy at the Crossroads. Gender and Welfare State Restructuring, London and New York : Routledge.

BRACHET S., (2001), «Politique familiale et assurance parentale en Suède : une synthèse », Dossier d'Étude, no 21, CNAF.

DAUNE-RICHARD A.-M., (1993), « Activité et emploi des femmes : des constructions sociétales différentes en France, au Royaume-Uni et en Suède », Sociétés contemporaines, $\mathrm{n}^{\mathrm{o}} 16$.

DAUNE-RICHARD A.-M., (1997), « Travail et citoyenneté : un enjeu sexué hier et aujourd'hui », in P. Bouffartigue, H. Eckert et J.-R. Pendariès, (ed.), Le travail à l'épreuve du salariat, L'Harmattan.

DAUNE-RICHARD A.-M., (1998a), « La garde des jeunes enfants en Suède. Un enjeu pour l'égalité des sexes », Cahiers du GEDISST, 22.

DAUNE-RICHARD A.-M., (1998b), «How dœs the "societal effect" shape the use of part-time work in France, the U.K. and Sweden », in J. O'Reilly and C. Fagan, (ed.), Part-time Prospects. An international comparison of part-time work in Europe, North America and the Pacific Rim, Routledge.

DAUNE-RICHARD A.-M., (1998c), «Qualifications et représentations sociales », in M. Maruani (sous la direction de), Les nouvelles frontières de l'inégalité. Hommes et femmes sur le marché du travail, La Découverte/MAGE.

DAUNE-RICHARD A.-M., (1999), « La notion de référentiel appliquée à la garde des jeunes enfants : une comparaison France-Suède », Recherches et Prévisions, 56, Paris, CNAF.

DAUNE-RICHARD A.-M., (à paraître), «Women's Work between Family and Welfare State : Part-time Work and Childcare in France and Sweden », in Pfau Effinger and Geissler, (ed.), Care Arrangements and Social Integration in European Societies.

GLUCKSMAN M., (1997), «L'organisation sociale globale du travail : une nouvelle approche pour une analyse sexuée du travail », Les Cahiers du Mage, 3-4.

JENSON J. et SINEAU M., (1998), «La France. Quand "liberté de choix" ne rime pas avec égalité républicaine », in Jenson et Sineau (sous la direction de), Qui doit garder le jeune enfant? Modes d'accueil et travail des mères dans l'Europe en crise, LGDJ, Paris.

KNIJN T. and KREMER M., (1997), « Gender and the Caring Dimension of Welfare States : Toward Inclusive Citizenship », Social Politics, 4 (3).

LETABLIER M.-T., (2001), « Le travail centré sur autrui et sa conceptualisation en Europe », Travail, genre et société, 6, p. 19-41.

LEWIS J., (2002), « Gender and Welfare State Change », European Societies, 4. 
MARSHALL T. H., (1950), Citizenship and Social Class, Cambridge University Press, Cambridge.

MELKAS H. et ANKER R., (1997), « Ségrégation professionnelle hommes-femmes dans les pays nordiques : une étude empirique », Revue internationale du travail, 3.

MULLER P., (1994), Les politiques publiques, PUF, « Que sais-je ? » $\mathrm{n}^{\circ} 2534$.

NYBERG A., (2000), «Les femmes sont-elles plus dépendantes de l'État providence que les hommes? », Cahiers du Genre, 27.

NYBERG A., (2002), « Gender, (de)commodification, economic (in)depedence and autonomous households : the case of Sweden », Critical Social Policy, 22 (1).

O’CONNOR J. S., (1993), « Gender, Class and Citizenship in the Comparative Framework of Welfare State Regimes : Theoretical and Methodological Issues », British Journal of Sociology, 44, 3.

O'CONNOR J. S., (1996), « From Women in the Welfare State to gendering Welfare State Regimes », Current Sociology, 44, 2.

ORLOFF A. S., (1993), « Gender and the social rights of citizenship : the comparative analysis of gender relations and welfare states », American Sociological Review, 58.

PERSSON I. et WADENSJÖ E., (2000), « À la recherche de l'égalité. Disparité salariale et division sexuelle du travail en Suède », Cahiers du Genre, 27.

SAINSBURY D., (1994), Gendering Welfare States, Sage, Londres.

VERJUS A., (2002), Le cens de la famille. Les femmes et le vote, 1789-1848, Belin, Paris.

WALBY S., (1994), «Is Citzenship Gendered ? », Sociology, 28/2. 